Review Paper http://ajol.info/index.php/ijbcs http://indexmedicus.afro.who.int

\title{
Synthèse des connaissances sur l'écologie et la biologie des crabes (Crustacés, Décapodes, Brachyoures)
}

\author{
Appolinaire GOUSSANOU ${ }^{1 *}$, Antoine CHIKOU ${ }^{2}$, Clément Abiola OGNI ${ }^{1}$, \\ Kévin Sagui KASSA ${ }^{1}$, Bernice HOUESSIONON ${ }^{1}$, Guy Apollinaire MENSAH ${ }^{3}$ et \\ Issaka YOUSSAO ABDOU KARIM ${ }^{1}$
}
${ }^{1}$ Laboratoire de Biotechnologie Animales et de Technologie des Viandes, Département de Production et Santé Animales, Ecole Polytechnique d'Abomey-Calavi, Université d'Abomey-Calavi, 01 BP 2009, Bénin.
${ }^{2}$ Laboratoire d'Hydrobiologie et d'Aquaculture, Faculté des Sciences Agronomiques, Université d'Abomey-Calavi, 01 BP 526 Cotonou, Bénin.
${ }^{3}$ Institut National des Recherches Agricoles du Bénin, Centre de Recherche Agricoles d'Agonkamey, 01 BP 884, Cotonou, Bénin.
*Auteur correspondant ; E-mail: goussanouappolinaire@ymail.com,iyoussao@yahoo.fr, Tel: 0022966153966 ;0022997912074.

\section{REMERCIEMENTS}

Les auteurs remercient le Ministère de l'Enseignent Supérieur et de la Recherche Scientifique du Bénin pour son soutien financier dans le cadre du projet de formation et d'appui aux doctorants.

\section{RESUME}

Les crabes représentent un groupe faunistique assez diversifiés et particulier en fonction du rôle bioécologique qu'ils jouent dans les écosystèmes aquatiques. Ces espèces sont de nos jours encore mal connues et méritent d'être étudiées dans le cadre de la mise en place des projets de gestions et de conservation. Cette revue fait le point sur les principaux traits liés à l'habitat, à la distribution et à la croissance de quelques espèces de crabes. Elle traite également et successivement des caractéristiques biologiques et écologiques de ces espèces tout en abordant les paramètres liés au cycle de reproduction, au régime alimentaire, à la fécondité et quelques aspects relatifs aux caractères morphologiques et aux relations de croissance chez ces espèces.

(C) 2017 International Formulae Group. All rights reserved.

Mots clés : Distribution, éco morphologie, alimentation, fécondité, croissance.

\section{Knowledge synthesis on the ecology and biology of crabs (Crustacea, Decapoda, Brachyura)}

\begin{abstract}
Crabs represent an enough diversified and particular faunistic group according to the bio-ecological role in the aquatics ecosystems. Currently we have little informations on these species, although they need to be studied in perspective for their use in management and conservation projects. This review summarizes the main traits related to the habitat, the distribution and the growth of some crab species. It also addresses the
\end{abstract}


aspects related to the biology and ecology of these species focusing on parameters such as reproductive cycle, fecundity, feeding strategies and morphological particularities.

(C) 2017 International Formulae Group. All rights reserved.

Keywords: Distribution, eco-morphology, feeding, reproduction, growth.

\section{INTRODUCTION}

Les crabes appartiennent au sous-ordre des Crustacés qui présentent une grande diversité si l'on considère leurs habitats, leurs physiologies, leurs structures et leurs comportements (D'Almeida et al., 2014 ; Olalekan et al., 2015). Ils sont largement distribués dans toutes les régions du globe et sont présents dans les habitats d'eaux douces, saumâtres et de mer. Les plus primitifs sont marins (Lawal-Are et Nwankwo, 2011). Certaines espèces par contre se rencontrent sur la terre ferme où ils peuvent vivre à des centaines de kilomètres des milieux aquatiques (D'Almeida et al., 2014 ; Vasquez et Ramirez, 2015). Les crabes comprennent plus de 6800 espèces et sous-espèces valides (Ng et al., 2008).

Les crabes jouent un rôle économique et bio-écologique important dans la plupart des écosystèmes aquatiques et occupent une grande variété de niches trophiques (Cartes et al., 2010). Certaines espèces sont comestibles et largement exploitées dans les écosystèmes aquatiques (Babatundé, 2008; Tohozin, 2012 ; Hinvi et al., 2013 ; Oluwatoyin et al., 2013 ; Sankaré et al., 2014a, 2014b). D'autres par contre, constituent des hôtes intermédiaires pour les parasites ou de la nourriture pour les oiseaux et d'autres organismes prédateurs (Cumberlidge, 2006 ; Tahir, 2012; Traoré, 2013). En plus de leurs rôles économiques et bioécologiques, la carapace de certaines espèces de crabes est largement recherchée et utilisée dans le domaine pharmaceutique à cause de leurs compositions riche en chitine. Cette carapace entre également dans la composition d'aliments pour poissons d'aquaculture et de volailles (Zouari, 2010).

En dépit du rôle biologique que joue ce groupe zoologique au sein des écosystèmes aquatiques, il faut signaler qu'ils ont fait l'objet de très peu d'études. Ils restent par conséquent encore mal connus du public et surtout de la communauté scientifique.

Cependant, dans les régions américaines et européennes, ces espèces ont déjà fait l'objet d'inventaires et de caractérisations bioécologiques. Quant aux espèces africaines, leurs études sont à leurs débuts. Les données disponibles sur ces espèces sont relatives aux travaux réalisées sur les crabes Callinectes amnicola, Callinectes pallidus Cardisoma armatum, Portunus validus, Carcinus aestuarii et le crabe de mangrove Scylla serrata (AkinOriola et al., 2005 ; Lawal-Are et Barakat, 2009; Nlewadim et al., 2009; Hountogan, 2011 ; Hinvi et al., 2013 ; Oluwatoyin et al., 2013 ; D'Almeida et al., 2014 ; Dessouassi, 2014 ; Sankaré et al., 2014a, 2014b ; Olalekan et al., 2015; Etchian et al., 2016). Les résultats de ces travaux méritent d'être connus dans le cadre de la mise en place des projets d'études, de gestions et de conservations de ces espèces. Ce travail de synthèse documentaire fait donc un état des connaissances disponibles sur la biologie et l'écologie des crabes d'eaux saumâtres, marines et de terre tout en se focalisant sur les aspects liés à leurs habitats, à leur croissance, à leur reproduction et à leur alimentation.

\section{TAXONOMIE DES CRABES}

Les crabes sont des invertébrés appartenant à l'embranchement des Arthropodes (corps et appendices articulés), au sous-embranchement des Antennates Mandibulates (présence d'antennes et de mandibule), à la classe des Crustacés (corps et appendices constitués de matière calcaire). Deux sous-groupes se dégagent de cette classe. Il s'agit des Entomostracés qui regroupent les Crustacés inférieures (de l'ordre $\mathrm{du} \mu \mathrm{m}$ ) et les Malacostracés qui 
regroupent les Crustacés supérieures. Les crabes appartiennent à la sous classe des Malacostracés, au super ordre des Eucarides (Crustacés aux yeux pédonculés dont la carapace couvre tout le thorax et est soudée à tous les segments), à l'ordre des Décapodes (possédant cinq paires de pattes), au sousordre des Reptantia (crustacés dont les appendices sont très développés et qui permettent la marche sur les fonds) et à la section des Brachyoures (crustacés décapodes dont l'abdomen est très réduit). En fonction de leurs habitats, de leurs modes de vie, de leurs structures et de leurs comportements, les crabes comptent plusieurs familles, genres et espèces (Bodin, 2005 ; Poupin, 2008).

\section{MORPHOLOGIE GENERALE}

Les crabes sont des animaux dont le corps, formé de la fusion de la tête et du thorax, est recouvert par une carapace dont la forme est variable suivant les espèces. Sur cet exosquelette, sont insérés des appendices ou pattes ambulatoires qui servent à la nage, à la marche, à la prise de la nourriture et surtout dans les parades. Certaines espèces de crabes sont adaptées exclusivement à la vie en eaux, d'autres par contre, mènent une existence terrestre mais sont largement dépendant du milieu aquatique pour une partie de leur cycle de vie (D'Almeida et al., 2014). Leur taille est très variable, comprise entre quelques millimètres, pour les crabes Cryptochiridae qui vivent dans les coraux, à près de $4 \mathrm{~m}$ d'envergure, pour le crabe géant Macrocheira kaempferi (Poupin, 2008). La Figure 1 présente quelques espèces de crabes.

\section{HABITAT ET DISTRIBUTION}

Les crabes colonisent tous les compartiments des milieux aquatiques. Ils sont présents dans les habitats d'eaux douces, d'eaux saumâtres et d'eaux de mer. On les rencontre dans les zones de mangrove, les plages de sables et même le milieu terrestre où ils peuvent vivre à des centaines de kilomètres des eaux (Bodin, 2005 ; Cumberlidge, 2006 ; Poupin, 2008). On les retrouve également dans les grandes profondeurs et s'adaptent à toutes les températures (Poupin, 2008).
Certaines espèces sont nageurs comme les Callinectes amnicola et les Portunus validus, d'autres par contre sont marcheurs comme les espèces du genre Cardisoma. Ces espèces appartiennent à un groupe faunistique largement distribué dans plusieurs pays du monde et d'Afrique (Cumberlidge, 2006; Guerrero et Arana, 2009; Poupin 2010; Mehmet, 2013 ; Vasquez et Ramirez, 2015). En Afrique, la présence de plusieurs espèces de crabes a été signalée en Côte d'Ivoire (D’Almeida et al., 2014; Sankaré et al., 2014a, 2014b; Etchian et al., 2016), au Nigéria (Babatundé, 2008; Lawal-Are et Barakat, 2009; Oluwatoyin et al., 2013; Olugbenga et Oloko, 2013; Omuvwie et Atobatelé, 2013; Olalekan et al., 2015), en Tunisie (Zouari, 2010), au Kenya (Fondo et al., 2010) et au Bénin (Hountogan, 2011 ; Hinvi et al., 2013 ; Dessouassi, 2014). Ils sont également présents dans de nombreux pays mais non documentés par la littérature.

\section{MUE ET CROISSANCE CHEZ LES CRABES}

La mue représente pour les crustacés un phénomène biologique important qui détermine la croissance. Du fait de la présence d'un squelette tégumentaire rigide et formé de matière calcaire, la croissance des crabes passe par des phases de mues successives au cours desquelles l'animal change sa carapace et tous les appendices calcifiés. La fréquence de la mue, sous contrôle nerveux et hormonale, varie avec l'espèce, l'âge, le sexe et les conditions du milieu (Miserey, 2005; Delaporte, 2007 ; Zouari, 2010). Le taux de croissance chez les crabes varie d'une espèce à l'autre et suivant les stades de développement (Zouari, 2010).

Le mécanisme de la mue n'a pas été suffisamment documenté chez les crabes. Cependant, les rares documents disponibles rapportent qu'elle débute par l'absorption d'une grande quantité d'eaux par l'animal, ce qui entraine un gonflement du corps, une élévation de la pression corporelle suivie d'une fragilisation de l'ancienne carapace. L'animal, par la suite, exerce des contractions musculaires stéréotypées ce qui provoque le 
décollement de l'ancien tégument (Miserey, 2005). Grâce à ces mouvements rythmiques, l'animal s'extrait de sa vieille carapace par l'existence des lignes de déhiscence. La mue encore appelée «exuvie » est abandonnée et la nouvelle carapace encore souple de même que les pièces internes encore molles s'épaississent et durcissent avec le temps grâce à l'absorption des substances chimiques (principalement le carbonate de calcium) contenu dans l'eau. Dans certains cas, notamment chez les crabes tropicaux et terrestres, Zouari (2010) signale que le durcissement rapide de la carapace résulte d'un dépôt de gastrolithes dans les tissus conjonctifs de la paroi de l'estomac avant la mue ou de la consommation de la quasitotalité de l'exuvie ce qui permet de récupérer les sels minéraux nécessaires au durcissement de la nouvelle carapace.

La durée de la mue varie d'une espèce à une autre. Elle peut durer plusieurs minutes sans toutefois excéder un quart d'heure chez les crabes, contrairement aux crevettes chez qui elle se déroule en quelques secondes seulement (Délaporte, 2007).

\section{AUTOTOMIE ET REGENERESCENCE DES ORGANES CHEZ LES CRABES}

L'autotomie représente un phénomène spécifique par lequel une espèce rejette un membre ou un appendice par une action réflexe. Cette capacité d'autotomie qui souvent observée chez les crustacés permet à ces espèces d'échapper à un prédateur en abandonnant l'appendice par lequel elles sont tenues (Miserey, 2005). Le mécanisme de l'autotomie chez les crustacés en particulier les crabes n'a pas été suffisamment documenté. Cependant, Zouari (2010) rapporte qu'il se déroule par la mise en place d'un caillot pelliculaire à la surface amputée ce qui empêche toute hémorragie lorsque l'animal abandonne l'appendice. Quelques jours après, un bourgeon de régénération apparaît au centre d'un plan d'autotomie. La durée d'apparition du bourgeon varie suivant la position de l'appendice et selon les stades biologiques. Le nouvel appendice qui fait son apparition est replié deux fois sur lui-même et enfermé dans un sac membranaire qui le protège extérieurement, grandit avec lui et reste mince et flexible tout au cours de la régénération (Zouari, 2010).

\section{REGIMES ALIMENTATIONS}

L'étude de l'alimentation reste souvent assez difficile puisqu'elle représente un premier pas dans la connaissance d'une espèce donnée. Etudier le régime alimentaire revient à établir et analyser l'ensemble des relations et interactions qui existent entre une espèce prédatrice et les diverses proies dont elle se nourrit. Chez les organismes aquatiques, l'analyse des contenus stomacaux a été effectuée afin d'identifier le choix des proies par espèces. Chez les espèces de crabes, les données concernant l'alimentation et l'analyse des contenus stomacaux restent très peu disponibles. Les travaux fondamentaux de Klassen et Locke (2007), Meynecke et al. (2010) mentionnent que les crabes sont en général des omnivores et des prédateurs. En effet, l'analyse des contenus stomacaux de quelques espèces révèle la présence de nombreux invertébrés et végétaux aquatiques comme les poissons, les mollusques, les crustacés, les vers annélides, les algues et même des grains de sable (Arimoro et Idoro, 2007; Lawal-Are et Barakat, 2009 ; Olalekan et Lawal-Are, 2013 ; Udoh et Ufonima, 2015) (Tableau 1). Certains auteurs signalent la présence dans les contenus stomacaux d'autres espèces de crabes ce qui indique une tendance cannibale chez ces espèces (Ryan, 2003 ; Lawal-Are et Barakat, 2009). Arimoro et Idoro (2007) signalent que chez Callinectes amnicola, les détritus représentent $58,38 \%$ de l'ensemble des proies consommées par l'espèce, suivi des crustacés $(30,36 \%)$, des poissons $(9,19 \%)$, des diatomées $(0,77 \%)$ et des algues filamenteuses $(1,33 \%)$.

\section{CYCLE DE REPRODUCTION}

La reproduction des crabes est sexuée. Les sexes sont séparés chez les crabes avec un dimorphisme sexuel remarquable. Toutefois, des cas d'hermaphrodisme sont signalés chez certaines espèces (Delaporte, 2007). 
L'identification du sexe chez les crabes est basée principalement sur la base de la forme de l'abdomen du mâle et de la femelle. Généralement, les femelles ont un abdomen circulaire ou ovalaire. Le cycle de reproduction chez les crabes qui sont des ovipares passe par des phases successives de maturation des organes génitaux, d'accouplement au cours de laquelle les ovules sont fécondés, de ponte, d'incubation des œufs et d'éclosion ou libération des larves (Zouari, 2010). L'accouplement a lieu généralement chez les crabes juste après la mue de la femelle. La fécondation est interne et après éclosion, les œufs libèrent une larve nauplius qui suit des phases successives de métamorphoses (segmentation de la région postérieure, différenciation des appendices, la dernière phase annonçant la différenciation sexuelle et la morphologique générale) (Delaporte, 2007 ; Zouari, 2010).

\section{FECONDITE CHEZ LES CRABES}

La fécondité exprime le pouvoir prolifique qui est l'une des stratégies qu'un organisme développe pour assurer sa pérennité. Elle correspond ici au nombre d'ovocytes mûrs présents dans l'ovaire immédiatement avant la ponte (Zouari, 2010). Chez les crabes, la fécondation est généralement interne. Les œufs fécondés issus de la fécondation sortent de la cavité au travers des gonopores et s'accumulent aux soies des pléopodes où ils entament leur croissance embryonnaire. Les femelles portant les grappes d'œufs fécondés sont des femelles ovigères ou «grainées ». Les études sur la fécondité chez les espèces de crabes ont été peu documentées. Les rares études ont été menées au Mexique, au Nigéria et en Tunisie. Chez Callinectes amnicola, la fécondité absolue varie entre 628 et 4.480 .500 oufs (Arimoro et Idoro, 2007; Lawal-Are, 2010 ; Olugbenga et Oloko, 2013). Selon ces auteurs, le nombre d'œufs portés par la femelle est fonction de la taille (largeur de la carapace) et $\mathrm{du}$ poids corporel. Ils signalent également qu'il existe une relation allométrique entre la fécondité et les variables morphologiques. Le diamètre des œufs chez $C$. amnicola mesure entre 0,25 et $0,35 \mathrm{~mm}$. Pour un total de 720 œufs dénombrés chez $C$. amnicola, Arimoro et Idoro (2007) rapportent un poids total de 8,99 g pour l'ensemble des œufs.

Chez Carcinus aestuarii, le nombre d'œufs portés par la femelle est en moyenne de 30.664 en Tunisie (Zouari, 2010) et de 272.162 en Turquie (Mehmet, 2013). Le diamètre moyen de l'œuf mesure $42010^{-3} \mathrm{~mm}$ (Mehmet, 2013). Il existe une corrélation positive d'une part entre le nombre d'œufs portée par la femelle et la largeur de sa carapace $\left(R^{2}=0,84\right)$ et d'autre part, entre ce dernier et le diamètre des œufs $\left(\mathrm{R}^{2}=0,94\right)$ (Mehmet, 2013).

Quant au Cardisoma crassum, la fécondité moyenne est de 501.022 œufs dans l'Estuaire El Salado au Mexique et le poids des œufs représente en moyenne 4,7\% du poids corporel de l'espèce (Vasquez et Ramirez, 2015).

Le nombre d'œufs porté par la femelle varie entre 806.000 et 6.240 .000 chez Portunus validus et le diamètre de l'œuf mesure en moyenne 0,34 $\mathrm{mm}$ (Lawal-Are et Barakat, 2009). Le Tableau 2 présente la fécondité absolue, le poids et le diamètre des œufs chez les crabes.

\section{CARACTERISTIQUES MORPHOMETRIQUES DES CRABES}

L'étude des variables morphométriques sur les ressources aquatiques est importante et trouve, en biologie des pêches, son application dans la gestion des pêcheries et dans l'évaluation des stocks halieutiques. Par des approches mathématiques, ces variables permettent de renseigner sur les conditions de vie des espèces aquatiques, leur croissance et leur survie, de même que leur maturité et reproduction (Abba et al., 2010; Chikou et al., 2011 ; Montcho, 2011 ; Montchowui et al., 2011 ; Niyonkuru et Lalèyè, 2012 ; Fagnon et al., 2013). Les principales mensurations souvent réalisées sur les crabes concernent le poids, la largeur et la longueur de la carapace. Le Tableau 3 présente la distribution des variables morphologiques mesurées chez certaines espèces de crabes. 
Les variables morphologiques rapportées chez les crabes par les différents auteurs varient d'une espèce à une autre et d'un milieu à un autre (Tableau 3). Il ressort de l'analyse de ce tableau que chez les espèces étudiées, Callinectes amnicola et Portunus validus ont présenté les plus grande taille (largeur de la carapace). Ensuite viennent les crabes Scylla serrata dont la largeur de la carapace varie entre 13,5 et $14,75 \mathrm{~cm}$ d'après les auteurs. Quant aux autres espèces, elles ont présenté des tailles relativement faibles. Ces différences du point de vue morphologique observées chez ces espèces peuvent être liées à plusieurs facteurs tels que les conditions physico-chimiques du milieu, la disponibilité alimentaire, les caractéristiques génétiques des espèces et les stress environnementaux.

\section{INDICES DE CROISSANCE CHEZ LES CRABES}

Chez les organismes vivants, la croissance massique ou métrique d'un individu ne suit pas toujours une croissance proportionnelle. L'étude de la croissance relative a été mise en évidence à travers un certain nombre d'indices biologique afin de traduire l'évolution des variables morphologiques des individus (Zouari, 2010). D'une façon générale, la relation exprimant l'évolution relative d'un organe ou d'une partie du corps par rapport à un caractère de référence est une fonction puissance de la forme $\mathrm{Y}=\mathrm{a} \mathrm{X}^{\mathrm{b}}$ où $\mathrm{Y}$ est le caractère étudié, $\mathrm{X}$ le caractère de référence, $b$ le coefficient d'allométrie ou de croissance et a la constante caractéristique de l'espèce. Le Tableau 4 présente les différentes relations entre les paramètres morphométriques et le poids chez les crabes.

Dans l'ensemble, la relation entre la largeur de la carapace et le poids chez les espèces étudiées indique que la croissance est de type allométrique négative $(b<3)$. Selon les différents auteurs, ceci s'observe généralement chez les espèces de crabes car leur croissance pondérale est moins importante que la croissance en taille. Toutefois, une croissance allométrique positive a été signalée chez certaines espèces en particulier le crabe Carcinus aestuarii (Mehmet, 2013).

Quant à la relation entre la longueur de la carapace et le poids, les différentes observations montrent que la majorité des espèces de crabes étudiées présentent également une croissance allométrique négative (Akin -Oriola et al., 2005 ; Arimoro et Idoro, 2007 ; Lawal-Are et Barakat, 2009 ; Olugbenga et Oloko, 2013 ; Mehmet, 2013 ; Olalekan et al., 2015) (Tableau 4). Cependant, Akin-Oriola et al. (2005) et Guerrero et Arana (2009) ont respectivement signalé une croissance isométrique chez Callinectes pallidus et Chaceon chilensis.

\section{PARASITISME ET EXPOSITION AUX METAUX TOXIQUES}

Les crabes font partie intégrante de l'environnement aquatique qui est de plus en plus contaminé par les pathogènes et les micros polluants. En fonction de leurs modes de vie et de leurs caractères fouisseurs, les crabes sont susceptibles d'accumuler d'importantes quantités de substances toxiques et d'être exposés aux parasites et autres symbiotes. Une synthèse de travaux réalisés par plusieurs auteurs est présentée dans le Tableau 5.

\section{IMPORTANCE BIO-ECOLOGIQUE ET AGRONOMIQUE DES CRABES}

Sur le plan écologique, les crabes jouent un rôle important dans le fonctionnement des écosystèmes aquatiques. Ils interviennent à différents niveaux trophiques comme herbivores, détritivores, prédateurs et même sous forme de proies (Cumberlidge, 2006). Certaines espèces constituent des hôtes intermédiaires pour certains parasites. C'est ainsi que dans certaines régions de l'Afrique de l'Ouest, il a été signalé que la consommation des crabes entraîne la paragonimiase chez l'homme car ils hébergent la douve du poumon (Paragonimus africanus et Paragonimus uterobilateralis qui ont été isolées en Afrique) (Cumberlidge, 2006 ; Traoré, 2013). Certaines espèces sont également utilisées comme 
espèces indicatrices ou biomarqueurs dans le cadre de l'évaluation de la qualité des milieux aquatiques par les métaux toxiques. C'est le cas du crabe vert européen Carcinus maenas (Brian, 2005; Stentiford et Feist, 2005 ; Moreira et al., 2006).

Comme les poissons et fruits de mer, les crabes constituent une importante source de protéines pour les populations riveraines de leur zone de prédilection, et parfois au-delà. Certaines espèces comme Callinectes amncola, Cardisoma armatum, Portunus validus et Scylla serrata, font l'objet d'une pêche active et d'une exploitation commerciale (Olalekan et Lawal-Are, 2013 ; Alberts-hubatsch, 2015 ; Etchian et al., 2016, etc.). Ils ont une valeur nutritionnelle intéressante. Ces espèces sont très recherchées et très appréciées pour la qualité de leurs chairs riches en minéraux et faibles en matière grasses (Trudeau, 2005). Ils constituent d'excellentes sources de zinc, de sélénium, de cuivre, de fer, de vitamine B12, B2 et B3, de magnésium et d'iode (Trudeau, 2005 ; Elegbede et Fashina-Bonbata, 2013 ; Traoré, 2013).

Notons aussi qu'il est important de souligner que les crabes comportent plusieurs espèces candidates à l'aquaculture. En Australie, de nombreuses études de domestication ont été réalisées sur les crabes du genre Scylla. Ces espèces possèdent de bons potentiels aquacoles et certaines sont bien élevées dans ce pays (Keenan et Blackshaw, 1999). Au Bénin, des essais de domestication de crabes Callinectes amnicola, Cardisoma armatum et Portunus validus ont été également réalisés (Hinvi et al., 2013; Edéa et al., 2015). Les résultats obtenus par ces auteurs à la fin des différents essais de croissance montrent de bonnes perspectives pour leur utilisation dans les systèmes aquacoles.

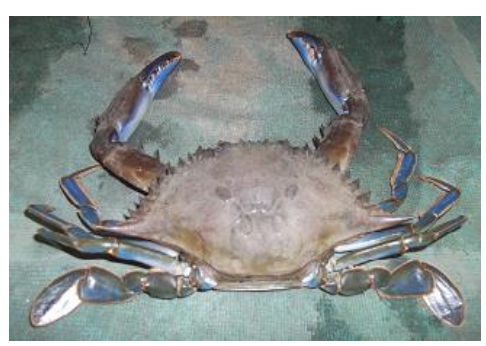

Callinectes amnicola

(Hountogan, 2011)

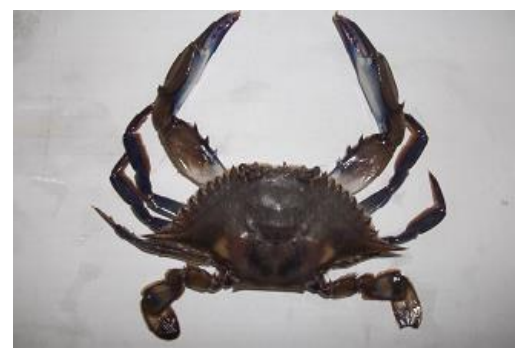

Portunus validus

(Hountogan, 2011)

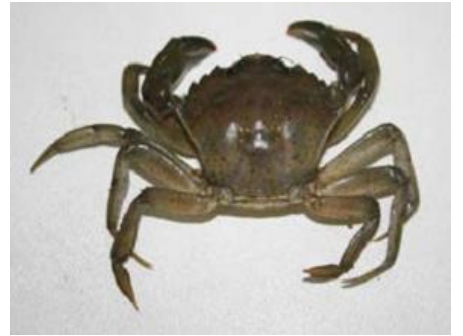

Carcinus aestuarii

(Zouari, 2010)

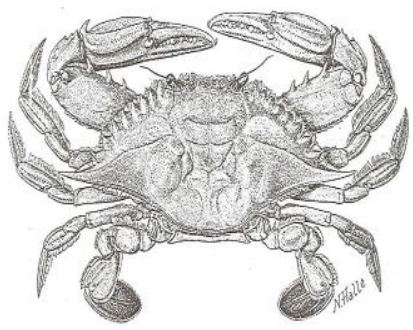

Callinectes pallidus

(Fischer et al., 81)

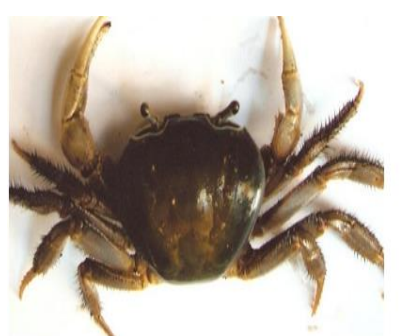

Cardisoma armatum

(Olalekan et Lawal-Are, 2013)

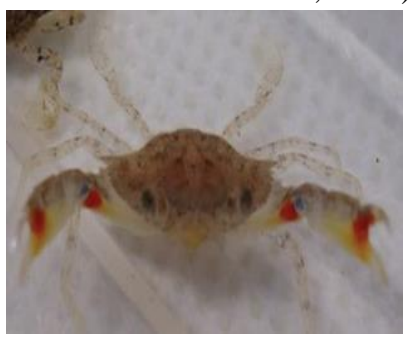

Scylla serrata

(Alberts-Hubatsch, 2015)

Figure 1 : Morphologie de quelques espèces de crabes. 
A. GOUSSANOU et al. / Int. J. Biol. Chem. Sci. 11(6): 2990-3004, 2017

Tableau 1 : Liste de quelques taxons (proies) consommés par Callinectes amnicola, Cardisoma armatum, Cardisoma guanhumi et Portunus validus.

\begin{tabular}{|c|c|c|c|c|c|}
\hline Espèces & Cours d'eaux & Plantes & Détritus & Animaux & Références \\
\hline & Warri (Nigéria) & algues, & grain de sable & poissons, crevettes, crabes & Arimoro et Idoro, 2007 \\
\hline Callinectes amnicola & $\begin{array}{l}\text { Rivières Qua Iboe et Imo } \\
\text { (Nigéria) }\end{array}$ & algues, plantes d'eaux & boue, grain de sable & $\begin{array}{l}\text { mollusques, crustacés, vers } \\
\text { polychètes, poissons, insectes, } \\
\text { nectons }\end{array}$ & Udoh et Ufonima ,2015 \\
\hline Cardisoma armatum & Zone de mangrove (Lagos) & $\begin{array}{l}\text { algues, idem, plantes } \\
\text { aquatiques }\end{array}$ & grains de sable, boue & poissons, crevettes, crabes, & \multirow{2}{*}{$\begin{array}{l}\text { Olalekan et Lawal-Are } \\
\text {,2013 }\end{array}$} \\
\hline Cardisoma guanhumi & Zone de mangrove (Lagos) & algues, & grain de sable, boue & poissons, crevettes, crabes, & \\
\hline Portunus validus & Côte de Lagos & algues & grain de sable & $\begin{array}{l}\text { annélides, crabes, crevettes, } \\
\text { gastéropodes, poissons, bivalves }\end{array}$ & $\begin{array}{l}\text { Lawal-Are et Barakat, } \\
2009\end{array}$ \\
\hline
\end{tabular}

Tableau 2 : Fécondité absolue, poids et diamètre des œufs chez les crabes.

\begin{tabular}{|c|c|c|c|c|c|}
\hline Espèces & Cours/Plan d'eaux & Poids des œufs (g) & Diamètres des œufs (mm) & Fécondité absolue & Références \\
\hline \multirow{3}{*}{ Callinectes amnicola } & Rivière Warri (Nigéria) & $7,11-10,89(8,99)$ & - & $628-812(720)$ & Arimoro et Idoro, 2007 \\
\hline & Lagune de Lagos (Nigéria) & - & $0,25-0,35(0,29)$ & $478.400-4.480 .500(780.480)$ & Lawal-Are, 2010 \\
\hline & Rivière Yewa (Nigéria) & - & - & $260.000-2.150 .692(126.9345)$ & Olugbenga et Oloko, 2013 \\
\hline \multirow[t]{2}{*}{ Carcinus aestuarii } & $\begin{array}{l}\text { Littoral Nord de Sfax } \\
\text { (Tunisie) }\end{array}$ & - & - & $3620-55.400(30.664)$ & Zouari, 2010 \\
\hline & Mer noir (Turquie) & $5,6-17,9(11,92)$ & $324,6-482(420) \times 10^{-3}$ & $179.899-375.820(272.162)$ & Mehmet, 2013 \\
\hline Cardisoma crassum & $\begin{array}{l}\text { Estuaire El Salado } \\
\text { (Mexique) }\end{array}$ & - & - & $173.595-866.857(501.022)$ & Vasquez et Ramirez, 2015 \\
\hline Portunus validus & Côte de Lagos (Nigéria) & - & $0,28-0,45(0,34)$ & $806.000-6.240 .000(2.200 .310)$ & Lawal-Are et Barakat, 2009 \\
\hline
\end{tabular}


A. GOUSSANOU et al. / Int. J. Biol. Chem. Sci. 11(6): 2990-3004, 2017

Tableau 3 : Distribution des variables morphologiques chez les espèces de crabes.

\begin{tabular}{|c|c|c|c|c|c|c|}
\hline Espèces & Pays & Cours/Plan d'eaux & Poids (g) & $\begin{array}{l}\text { Largeur de la } \\
\text { carapace }(\mathrm{cm})\end{array}$ & $\begin{array}{c}\text { Longueur de la } \\
\text { carapace }(\mathrm{cm})\end{array}$ & Références \\
\hline \multirow{8}{*}{ Callinectes amnicola } & \multirow{5}{*}{ Nigéria } & Rivière Warri & $23,0-107,8$ & $7,5-11,4$ & $3,5-7,5$ & Arimoro et Idoro, 2007 \\
\hline & & Rivière Imo & $4,0-307,0$ & $4,3-16,64$ & $2-8,1$ & Udoh et al., 2009 \\
\hline & & Rivière Okpoka & $1,17-151,62$ & $1,1-7,6$ & - & Abowei et George, 2010 \\
\hline & & Rivière Yewa & $8,2-165,00(74,16)$ & - & $5,2-14,8(10,75)$ & Olugbenga et Oloko, 2013 \\
\hline & & Lagune de Lagos (Badore, Ajah) & $19,80-190,3(109,93)$ & $7,5-15,00(12,26)$ & $3,50-7,00(5,61)$ & Omuvwie et Atobatelé, 2013 \\
\hline & Bénin & Lac Ahémé & 65,17 & $4,31-17,45(9,50)$ & - & Dessouassi, 2014 \\
\hline & \multirow{2}{*}{ Nigéria } & Rivière Qua Iboe & 118,04 & - & 6,57 & \multirow{2}{*}{ Udoh et Ufonima, 2015} \\
\hline & & Rivière Imo & 137,50 & - & 6,37 & \\
\hline \multirow{2}{*}{ Callinectes pallidus } & Nigéria & Rivière de Badagri & $10,7-220,20(126,27)$ & - & $3,80-6,50(5,17)$ & Akin - Oriola et al., 2005 \\
\hline & Nigéria & Rivière Ojo & $26,0-143,4(74,44)$ & $3,2-6,3(4,83)$ & $2,8-6,00(4,81)$ & Oluwatoyin et al., 2013 \\
\hline Carcinus aestuarii & Turquie & Mer noir & $0,76-172(74,38)$ & $1,25-9,2(6,35)$ & $1,0-7,0(5,02)$ & Mehmet, 2013 \\
\hline \multirow{2}{*}{ Cardisoma armatum } & \multirow{2}{*}{ Nigéria } & Rivière de Badagri & $38,07-214,60(110,2)$ & - & $3,20-5,80(4,38)$ & Akin - Oriola et al., 2005 \\
\hline & & Lagune de Lagos & $9,6-290,00$ & $2,70-9,40$ & $2,50-9,30$ & Olalekan et al., 2015 \\
\hline Cardisoma crassum & Mexico & Estuaire El Salado & 125,87 & 5,68 & - & Vazquez et Ramirez, 2015 \\
\hline Cardisoma guanhumi & Nigéria & Lagune de Lagos & $4,70-295,00$ & $2,80-9,40$ & $2,50-9,20$ & Olalekan et al., 2015 \\
\hline Chaceon chilensis & Chili & Archipel Juan Fernandez & & & 10,66 & Guerrero et Arana, 2009 \\
\hline Portunus validus & Nigéria & Côte de Lagos & $82,3-694,0$ & $9,2-19,5$ & $4,5-11,4$ & Lawal-Are et Barakat, 2009 \\
\hline \multirow{3}{*}{ Scylla serrata } & Kenya & Malindi & - & - & $5,00-12,5$ & Fondo et al., 2010 \\
\hline & Iran & Golfe Persique et d'Oman & 686,45 & 14,72 & - & $\begin{array}{l}\text { Rezaie-Atagholipour et al., } \\
2012\end{array}$ \\
\hline & Australie & Queensland & - & 13,5 & - & Alberts - Hubatsch, 2015 \\
\hline
\end{tabular}


A. GOUSSANOU et al. / Int. J. Biol. Chem. Sci. 11(6): 2990-3004, 2017

Tableau 4 : Paramètres d'estimation de la relation largeur de la carapace - poids, longueur de la carapace - poids et type de croissance chez les crabes

\begin{tabular}{|c|c|c|c|c|c|c|c|c|c|c|}
\hline \multirow{2}{*}{ Espèces } & \multirow{2}{*}{$\begin{array}{l}\text { Cours/Plan } \\
\text { d'eaux }\end{array}$} & \multicolumn{4}{|c|}{$\mathrm{W}=\mathbf{a} \operatorname{largC} C^{b}$} & \multicolumn{4}{|c|}{$\mathrm{W}=\mathbf{a}$ long $\mathrm{C}^{\mathrm{b}}$} & \multirow{2}{*}{ Référence } \\
\hline & & $\mathbf{a}$ & b & $\mathbf{r}^{2}$ & TC & $\mathbf{a}$ & b & $\mathbf{r}^{2}$ & TC & \\
\hline \multirow{5}{*}{ Callinectes amnicola } & Rivière Warri & & & & & 1,719 & 2,414 & 0,1279 & A- & Arimoro et Idoro, 2007 \\
\hline & Lagune de Lagos & & & & & $-1,342$ & 3,1784 & 0,9756 & $\mathrm{~A}+$ & Babatundé, 2008 \\
\hline & Rivière Okpoka & $-2,373$ & 2,449 & 0,961 & A- & & & & & Abowei et George, 2009 \\
\hline & Lagune de Lagos & $-1,05$ & 2,81 & 0,83 & A- & $-0,33$ & 3,12 & 0,82 & $\mathrm{~A}+$ & Omuvwie et Atobatelé, 2013 \\
\hline & Lac Ahémé & 0,128 & 2,706 & 0,954 & A- & & & & & Dessouassi, 2014 \\
\hline Callinectes pallidus & Rivière de Badagri & & & & & $-0,18$ & 3,01 & 0,35 & I & Akin -Oriola et al., 2005 \\
\hline Carcinus aestuarii & Mer noir & 0,213 & 3,117 & 0,954 & $\mathrm{~A}+$ & 0,574 & 2,954 & 0,97 & A- & Mehmet, 2013 \\
\hline \multirow{2}{*}{ Cardisoma armatum } & Rivière de Badagri & & & & & 2,42 & 1,50 & 0,19 & A- & Akin -Oriola et al., 2005 \\
\hline & Lagune de Lagos & 2,1239 & 0,1843 & 0,1434 & A- & 1,8777 & 0,3378 & 0,2046 & A- & Olalekan et al., 2015 \\
\hline Cardisoma crassum & Estuaire El Salado & 0,36 & 2,55 & 0,863 & A- & & & & & Vasquez et Ramirez, 2015 \\
\hline Cardisoma guanhumi & Lagune de Lagos & 1,8927 & 0,2383 & 0,0698 & A- & 1,9095 & 0,21125 & 0,0577 & A- & Olalekan et al., 2015 \\
\hline Chaceon chilensis & $\begin{array}{l}\text { Archipel Juan } \\
\text { Fernandez }\end{array}$ & & & & & 0,0004 & 3,069 & 0,99 & I & Guerrero et Arana, 2009 \\
\hline Portunus validus & Côte de Lagos & & & & & $-0,933$ & 2,857 & 0,889 & A- & Lawal-Are et Barakat, 2009 \\
\hline
\end{tabular}

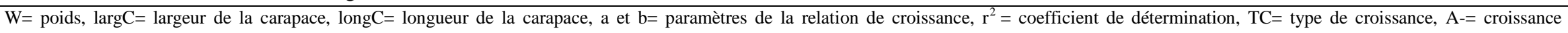
allométrique négative, $\mathrm{A}+=$ croissance allométrique positive, $\mathrm{I}=$ croissance isométrique 
Tableau 5 : Parasites et métaux toxiques dans les espèces de crabes

\begin{tabular}{llll}
\hline Espèces & Microorganismes & Parasites & Métaux toxiques \\
\hline $\begin{array}{l}\text { Callinectes } \\
\text { amnicola }\end{array}$ & $\begin{array}{l}\text { Coliformes fécaux, Bactéries } \\
\text { anaérobies sulfito réductrices, } \\
\text { Salmonelles, Clostridium } \\
\text { perfringens, Vibrio (Koussémon } \\
\text { al., 2008, Traoré, 2013) }\end{array}$ & $\begin{array}{l}\text { Ectoparasites } \\
\text { (Omuwvie et } \\
\text { Atabatelé, 2013) } \\
\text { Trématodes } \\
\text { (Traoré, 2013) }\end{array}$ & $\begin{array}{l}\text { Manganèse, Zinc, Plomb, Nickel, fer, } \\
\text { Cadmium, Mercure (Oyebisi et al., } \\
\text { 2012; Omuwvie et Atobatelé, 2013; } \\
\text { Gbogbo et al., 2016) }\end{array}$ \\
\hline $\begin{array}{l}\text { Cardisoma } \\
\text { armatum }\end{array}$ & - & $\begin{array}{l}\text { Trématodes } \\
\text { (Traoré, 2013) }\end{array}$ & $\begin{array}{l}\text { Fer, Zinc, Nickel, Cadmium, Mercure, } \\
\text { Plomb (Oyebisi et al., 2012, Gbogbo et } \\
\text { al., 2016) }\end{array}$ \\
\hline $\begin{array}{l}\text { Carcinus } \\
\text { aestuarii }\end{array}$ & - & $\begin{array}{l}\text { Sacculine } \\
\text { (Zouari, 2010) }\end{array}$ & \\
\hline $\begin{array}{l}\text { Portunus } \\
\text { segnis }\end{array}$ & - & $\begin{array}{l}\text { Chelonibia } \\
\text { platula } \text { (Tahir, } \\
\text { 2012) }\end{array}$ & \\
\hline $\begin{array}{l}\text { Portunus } \\
\text { pelagicus }\end{array}$ & - & - & $\begin{array}{l}\text { Cadmium, Arsenic (Fatemi et } \\
\text { Shahrzad, 2016) }\end{array}$ \\
\hline $\begin{array}{l}\text { Trichodactylis } \\
\text { fluviatilis }\end{array}$ & - & \multicolumn{1}{c}{ - } & Cadmium et Plomb (Franchi et al, \\
\hline
\end{tabular}

\section{Conclusion}

Ce travail de synthèse réalisé sur les crabes a permis de faire un bilan des connaissances disponibles sur ces espèces. Au total, 9 espèces aussi bien tropicales, tempérées que d'eaux saumâtres, marines et de terre ont fait l'objet d'inventaires et d'analyses. Les crabes sont des espèces omnivores et prédatrices qui exploitent une large gamme de proies. Ils s'adaptent à différents types d'habitats allant du milieu aquatique à la terre ferme. La croissance chez les crabes s'effectue par mue successive et la reproduction passe par des phases successives de maturation des gonades, d'accouplement, de ponte, d'incubation et d'éclosion. La fécondité est caractéristique de chaque espèce et varie en fonction de la taille et du poids des individus. L'évolution des variables morphologiques est caractéristique de l'espèce et du milieu et se traduit par différentes forme de croissance.

\section{CONFLIT D'INTERETS}

Les auteurs déclarent qu'ils n'ont aucun conflit d'intérêts.

\section{CONTRIBUTION DES AUTEURS}

AG a effectué la recherche documentaire et rédaction de l'article de synthèse. $\mathrm{AC}$ a effectué la correction et les amendements de la synthèse sur la taxonomie et la morphologie des crabes. CAO a effectué la correction et les amendements de la synthèse sur l'habitat, la distribution, la mue et la croissance des crabes. KSK a effectué la correction et l'amendement de la synthèse sur le cycle de reproduction et la fécondité chez les crabes $\mathrm{BH}$ a effectué la correction et l'amendement de la synthèse sur les caractéristiques morphométriques et les indices de croissance chez les crabes. GAM a effectué la correction et l'amendement de la synthèse sur le parasitisme et l'importance bio-écologique et agronomique des crabes. IYAK a été le superviseur général de la rédaction de l'article de synthèse et validation de l'article après sa rédaction.

\section{REMERCIEMENTS}

Les auteurs remercient l'équipe de recherche du Laboratoire de Biotechnologie Animale et de Technologie des Viandes de l'Ecole Polytechnique d'Abomey-Calavi et 
son Directeur le Professeur Issaka YOUSSAO ABDOU KARIM pour leurs différentes contributions.

\section{REFERENCES}

Abba E, Belghyti D, Benabid M, El Ibaoui H. 2010. Relation taille-poids et coefficient de condition de la truite commune (Salmo trutta macrostigma dumeril, 1858) de l'Ouest Sidi Rachid (Moyen Atlas) Maroc. Afrique Sciences, 6(2): 60-70.

Abowei JFN, George ADI. 2009. A study of length-weight relationship and condition factor of Callinectes amnicola (De Rochebrune, 1883) from Opkoka Creek, Niger Delta, Nigeria. Int. J. Anim. Veter. $A d v ., 1(2):$ 66-72.

Abowei JFN, George ADI. 2010. The morphology, abundance, size and sex distribution of Callinectes amnicola (De Rochebrune, 1883) from Okpoka Creek, Niger Delta, Nigeria. Curr. Res. J. Biol. Sci., 2(1): 27-34.

Akin-Oriola G, Anetekhai M, Olowonirejuaro K. 2005. Morphometric and meristic studie in two crabs: Cardisoma armatum and Callinectes pallidus. Turk. J. Fish. Aquat. Sci., 5: 85-89.

Alberts-Hubatsch H. 2015. Movement patterns and habitat use of the exploited swimming crab Scylla serrata (Forskal, 1775). Dissertation in partial fulfillment of the requirements for the degree of Doctor of Natural Sciences. Faculty of Biology/Chemistry, University Bremen, Germany, p. 129.

Arimoro FO, Idoro B. 2007. Ecological studies and biology of Callinectes amnicola (Family: Portunidae) in the Lower Reaches of Warri River, Delta State, Nigeria. World J. Zool., 2(2): 57-66.

Babatunde EE. 2008. The fishery and bionomics of the swimming crab, Callinectes amnicola (De Rochebrune, 1883) from a Tropical Lagoon and its Adjacent Creek, Southwest, Nigeria. J. Fish. Aquat. Sci., 3(2): 114-125.

Bodin N. 2005. Contamination des crustacés décapodes par les composés organohalogénés. Etude détaillée de la bioaccumulation des PCB chez l'araignée de mer Maja brachydactyla. Thèse de Doctorat de l'Université de Bretagne Occidentale, Région Bretagne, p. 328

Brian JP. 2005. Inter- population variability in the reproductive morphology of the shore crab (Carcinus maenas): evidence of endocrine disruption in a marine crustacean? Mar. Pollut. Bull., 50: 410416.

Cartes JE, Fanelli E, Papiol V, Maynou F. 2010. Trophic relationships at intrannual spatial and temporal scales of macro and megafauna around a submarine canyon of the Catalonian coast (western Mediterranean). J. Sea Res., 63:180-190.

Chikou $\mathrm{A}$, Laleyè $\mathrm{P}$, Bonou $\mathrm{C}$, Vandewalle $\mathrm{P}$, Philippart JC. 2011. Tailles de première maturité et de capture de six espèces de poissons-chat dans le delta de l'Ouémé au Bénin (Afrique de l'ouest). Int. J. Biol. Chem. Sci., 5(4) 1527-1537. DOI : http://dx.doi.org/10.4314/ijbcs.v5i4.18.

Cumberlidge N. 2006. Description des espèces de crustacés collectées dans le Nord-Ouest de la Guinée: Rapid Assessment Program (RAP). Bulletin of Biological Assessment, 41, Annexe 3, p. 168-175, Conservation International, Washington DC.

D'Almeida MA, Koua HK, Ouattara-Soro SF, Fantodji A, Ehouman A, N'Diaye AS. 2014. Caractères généraux et étude histologique du développement embryonnaire $\mathrm{du}$ crabe terrestre, Cardisoma armatum, herklots, 1851 (decapoda gecarcinidae) de Côté d'Ivoire. Afrique Science, 10(2) : 338-355.

Delaporte G. 2007. La biologie sous-marine : les crustacés, p. 10.

Dessouassi CE. 2014. Exploitation du crabe Callinectes amnicola (De Rochebrune, 1883) au lac Ahémé (Sud-Bénin) et données préliminaire sur la biologie de l'espèce. Mémoire présenté pour l'obtention du grade de master spécialisé en aménagement des pêches et aquaculture, Faculté des Sciences Agronomiques, Université d'AbomeyCalavi, p. 86. 
Edéa OG, Gbangboché AB, Hinvi LC, Azando EV. 2015. Breeding of the land crab Cardisoma armatum (Herklots) in enclosure in Benin. J. Appl. Biosci., 96: $9102 \quad-\quad 9108 . \quad$ DOI: http://dx.doi.org/10.4314/jab.v96i1.7.

Elegbede IO, Fashina-Bombata HO. 2013. Proximate and Mineral compositions of common crab species (Callinectes pallidus and Cardisoma armatum) of Badagry Creek, Nigeria. Poult. Fish. Wildl. Sci., 2(1): $1-5 . \quad$ DOI: http://dx.doi.org/10.4172/pfw.1000110

Etchian OA, Dakouri R, Blé CM, Lawal-Are AO, Cuesta AJ. 2016. Some ecological aspects of the gecarcinid land crab, Cardisoma armatum Herklots, 1851 (Crustacea, Brachyura, Gecarcinidae) from the estuarine region of the Comoe River, Côte d'Ivoire. Int. J. Biol. Chemi. Sci., 10(2): $\quad 457-474 . \quad$ DOI http://dx.doi.org/10.4314/ijbcs.v10i2.1

Fagnon SM, Chikou A, Youssao I, Laleyè P. 2013. Caractérisation morphologique des populations de Sarotherodon melanotheron (Pisces, Cichlidae) en eaux douces et saumâtres au Sud Bénin. Int. J. Biol. Chem. Sci., 7(2) : 619-630.

Fatemi F, Shahrzad K. 2016. Investigation of cadmium and arsenic accumulation Portunus pelagicus along the Asalouyeh Coast, Iran. Journal of Earth, Environment and Health Sciences, 2(1): 34-38. DOI: 10.4103/2423-7752.181805

Fondo EN, Kimani EN, Odongo D. 2010. The status of mangrove mud crab fishery in Kenya East Africa. Int. J. Fish. Aquac., 2(3): 79-86.

Franchi M, Menegario A, Brossi-Garcia A, Chagas CG, Silva MV, Antonio CSP, Govone JS. 2011. Bioconcentration of cadmium and plomb by the Rivers crab Trichodactylus fluviatilis (Crustacea: Decapoda). J. Braz. Chem. Soc., 22(2): 230-238.

Gbogbo F, Otoo SD, Huago RQ, Asomaing O. 2016. High levels of mercury in wetland resources from three river basins in Ghana: a concern for public health. Environ. Sci. Pollut. Res., p. 9. DOI 10.1007/s11356-
016-8309-2. DOI $10.1007 / \mathrm{s} 11356-016-$ 8309-2.

Guerrero A, Arana P. 2009. Size structure and sexual maturity of the golden crab (Chaceon chilensis) exploited off Robinson Crusoe Island, Chile. Lat. Am. J. Aquat. Res., 37(3): 347-360. DOI: 10.3856/vol37-issue3-fulltext-6

Hinvi LC, Sohou Z, Agadjihouedé H, Laleye P, Sinsin B. 2013. Domestication de Portunus validus et Callinectes amnicola au Bénin. J. Rech. Sci. Univ. Lomé (Togo), série (A), 15(2) : 13-22.

Hountogan M. 2011. Diversité et exploitation des crabes du Lac Nokoué au Bénin. Mémoire présenté pour l'obtention du grade de master professionnel en gestion des ressources naturelles et de la biodiversité, Faculté des Sciences Agronomiques, Université d'AbomeyCalavi, p. 86.

Keenan CP, Blackshaw A. 1999. Mud crab aquaculture and biology. Proceedings of an international scientific forum held in Darwin, Australia, No 78, p. 216.

Klassen G, Locke, A. 2007. Synthèse de la biologie du crabe vert européen (Carcinus maenas). Rapport manuscrit Canadien des sciences halieutiques et aquatiques, 2818 F : vii + p. 82.

Koussémon M, Traoré SG, Koffi-Nevry R, Ouffoue SK, Kamenan A. 2008. Etude la qualité microbiologique d'une espèce tropical de crabe: Callinectes amnicola. Tropicultura, 26(3) : $169-172$.

Lawal-Are AO. 2010. Reproductive biology of the blue crab, Callinectes amnicola (De Rochebrune) in the Lagos Lagoon, Nigeria. Turk. J. Fish. Aquat. Sci., 10: 1-7. DOI: 10.4194/trjfas.2010.0101

Lawal-Are AO, Barakat B. 2009. The biology of the smooth swim crab, Portunus validus (Herklots) off Lago's Coast, Nigeria. European Journal of Scientific Research, 30(3): 402-408.

Lawal-Are AO, Nwankwo H. 2011. Biology of the hairy mangrove crab, Sersema huzardii (Decapoda: Graspidae) from a tropical Estuarine Lagoon. J. Am. Sci., 7: 45-48. 
Mehmet A. 2013. Length-Weight relationship and reproductive features of the Mediterranean Green crab, Carcinus aestuarii Nardo, 1847 (Decapoda: Brachyura) in the Eastern Black Sea, Turkey. Pakistan J. Zool., 45(6): 16151622.

Meynecke JO, Shing YL, Grubert M, Brown I, Steven M, Gribble N, Johnston D, Gillson J. 2010. Evaluating the environmental drivers of mud crab (Scylla serrata) catches in Australia. Final Report FRDC Project 2008/012, p. 6.

Miserey B. 2005. Les crustacés : caractéristiques et morphologie externe. Commission Départementale de Biologie sous-marine de l'Oise, p. 7.

Montcho SA. 2011. Diversité et exploitation des poissons de la Rivière Pendjari (Bénin, Afrique de l'Ouest). Thèse du grade de Docteur de l'université d'Abomey-Calavi (Bénin). p. 234.

Montchowui E, Ovidio M, Laleyè P, Philippart JC, Poncin P. 2011. Stratégies de reproduction des populations chez Labeo parvus Boulenger, 1902 (Cypriniformes: Cyprinidae) dans le bassin du fleuve Ouémé au Bénin. Annales des Sciences Agronomiques, 15(2) : 153-171.

Moreira SM, Guilhermino L, Ribeiro R. 2006. An in situ postexposure feeding assay with Carcinus maenas for estuarine sedimentsoverlying water toxicity evaluations. Environmental Pollution, 139: 318-329.

Ng Peter KL, Guinot D, Davie PJF. 2008. Systema Brachyurorum: Part I. An annotated checklist of extant Brachyuran crabs of the world. The Raffles Bulletin of Zoology, 17:1-286.

Niyonkuru C, Laleyè P. 2012. A comparative ecological approach of the length-weight relationships and condition factor of Sarotherodon melanotheron Rüppell, 1852 and Tilapia guineensis (Bleeker, 1862) in Lake Nokoué and Ahémé (Bénin, West Africa). Int. J. Busi. Huma. Tech., 2(3): 41-50.

Nlewadim AA, Ofor CO, Udoh JP. 2009. Size composition and population characteristics of the swimming crab Callinectes amnicola (De Rochebrune, 1883) (Crustacea, Brachyura, Portunidae) in the Imo River Estuary, Nigeria. Nigeria Journal of Agriculture, Food and Environment, 5(2-4): 47-60.

Olalekan EI, Lawal-Are AO. 2013. Comparative study of condition factor, stomach analysis and some aspects of reproductive biology of two land carbs: Cardisoma armatum (Herklots, 1851) and Cardisoma guanhumi (Latreille, 1825) from a mangrove swamp ecosystem, Lagos, Nigeria. J. Marine Sci. Res. Dev., 4(1): $\quad 1-4$. DOI: $10.4172 / 2155-$ 9910.1000143.

Olalekan EI, Lawal-Are AO, Titilade PR. 2015. Size and growth of Cardiosoma armatum and Cardisoma guanhumi as ecological parameters for mangrove ecosystem. J. Marine Sci. Res. Dev., 5(2): 1-7. DOI: http://dx.doi.org/10.4172/21559910.1000164

Olugbenga E, Oloko LRT. 2013. Growth patterns, sex ratio and fecundity estimates in blue crab (Callinectes amnicola) from Yewa River, Southwest Nigeria. Advances in Life Sciences and Technology, 7: 24-34.

Oluwatoyin A, Akintade A, Edwin C, Kusemiju V. 2013. A study of lengthweight relationship and condition factor of West African blue crab (Callinectes pallidus) from Ojo Creek, Lagos Nigeria. American Journal of Research Communication, 1(3): 102-114.

Omuvwie U, Atobatele O. 2013. Growth pattern condition factor, trace metal studies and ectoparasitic load of the blue crab, Callinectes amnicola from Lagos Lagoon, Badore, Ajah, Lagos, Nigeria. Cameroon Journal of Experimental Biology, 9(1): 34-43. DOI: http://dx.doi.org/10.4314/cajeb.v9i1.5.

Oyebisi R, Lawal-Are A, Alo B. 2012. Comparative study of persistent toxic metals levels in land crab (Cardisoma armatum) and lagoon crab (Callinectes amnicola) in Lagos Lagoon. Scholarly J. Biotechnol., 1(4): 72-79.

Poupin J. 2008. Crustacés de l'île de la Réunion (Décapodes et Stomatopodes). 
Rapport scientifique préliminaire de l'Institut de Recherches de l'Ecole Navale, p. 85, figures 1-35.

Poupin J. 2010. Biodiversité de l'IndoPacifique tropical français. Rapport scientifique de l'Institut de Recherche de l'Ecole Navale, p. 80.

Rezaie-Atagholipour M, Naderloo R, Kamrani E, Savari R. 20120. Preliminary biological information of Scylla serrata (Forskal, 1775) (Brachyura, Portunidae) in the Persian Gulf and Gulf of Oman: A conservation priority. Crustaceana 86(3): 322-335.

Ryan S. 2003. Ecological assessmentqueensland mud crab fishery. Queensland fisheries service, Department of Primary Industries, Brisbane, p. 36.

Sankaré Y, Amalatchy NJ, Koffie-Bikpo CY. 2014a. Etude comparative des captures de crabes nageurs Callinectes amnicola (Decapoda, Portunidae) dans les lagunes Ivoiriennes (Afrique de l'Ouest). Rev. CAMES, 2(1) : 75-84.

Sankaré Y, Konan KJ, Amalatchy NJ., Soro MB. 2014b. Swimming crab Callinectes amnicola (Decapoda-Portunidae): capture analysis of a Lagoon with high continental influence (Aby Lagoon, Côte d'Ivoire, West Africa). Bio. Sci., 2(2): 9-19.

Stentiford GD, Feist SW. 2005. A histopathological survey of shore crab (Carcinus maenas) and brown shrimp (Crangon crangon) from six estuaries in the United Kingdom. Journal of Invertebrate Pathology, 88: 136-146.

Tahir Ö. 2012. The swimming crab Portunus segnis (Forskâl, 1775): host for the barnacle Chelonibia platula (Ranzani, 1818) from the Turkish coast. J. Black Sea/Mediterranean Environment, 18(3): 271-278.

Tohozin YA. 2012. Pêcheries sédentaires, production et commercialisation des tourlourous des lagunes (Cardisoma armatum), des crabes bicornes (Callinectes amnicola) et des étrilles lisses (Portunus validus) dans l'ouest du lac Nokoué en République du Bénin. J. Rech. Sci. Univ. Lomé, 14(1) : 12 -21.

Traoré SG. 2013. Risques de contraction des affections à Vibrio sp. et à Paragonimus sp. liés à la consommation des crabes et des crevettes vendus sur les marchés d'Abidjan et de Dabou. Thèse pour l'obtention du grade de Docteur en Sciences et Technologies des Aliments de l'Université Nangui Abrogoua, p. 198.

Trudeau C. 2005. Santé Canada: Fichier canadien sur les éléments nutritifs.

Udoh JP, Nlewadim AA, Ofor C. 2009. Maturity estimation in male swimming brachyuran crab, Callinectes amnicola (De Rochebrune, 1883) (Decapoda, Portunidae) in the Imo River Estuary, Nigeria. Nigeria Journal of Agriculture, Food and Environment, 5(2-4): 61-71.

Udoh JP, Ufonima UJ. 2015. Dietary spectrum, dispersity and overlaps of blue crab (Callinectes amnicola, De Rochebrune) from Southeast Nigeria. Croatian Journal of Fisheries, 73: 162169. DOI: http://dx.doi.org/10.14798/73.4.852.

Vasquez LH, Ramirez PT. 2015. Aspects of growth in the terrestrial crab Cardisoma crassum Smith, 1870 (Crustacea: Brachyura: Gecarcinidae) from El Salado Estuary Puerto Vallarta, Jalisco, Mexico. Research Gate, Mitteilungen Klosterneuburg, 65(2): 81-99.

Zouari SB. 2010. Contribution à l'étude écobiologique et biochimique du crabe Carcinus aestuarii dans la région Nord de Sfax. Mémoire présenté pour l'obtention du diplôme de mastère en biodiversité et ressources aquatiques, Faculté des Sciences de Sfax, Université de Sfax, p. 104. 